\title{
HAUSDORFF MEASURE AND CARLESON THIN SETS ${ }^{1}$
}

\author{
JOEL H. SHAPIRO
}

\begin{abstract}
A Carleson set is a closed subset of the unit circle $T$ having measure zero, whose complement is a disjoint union of open subarcs $\left(I_{n}\right)$ with $\Sigma\left|I_{n}\right| \log \left|I_{n}\right|>$ $-\infty$. Suppose $H$ is the Hausdorff measure on $T$ induced by the determining function $h$, where $h(t) / t$ is strictly decreasing. We show that $H(E)=0$ for every Carleson set $E$ if and only if $\int_{0}^{1} h(t)^{-1} d t=\infty$. Consequently the nonintegrability of $h^{-1}$ is necessary and sufficient for every positive Borel measure $\mu$ on $T$ with modulus of continuity $\omega_{\mu}(\delta)=O(h(\delta))$ to place zero mass on every Carleson set.
\end{abstract}

1. Introduction. Suppose $E$ is a closed subset of the unit circle $T$, so its complement $T \backslash E$ can be written as a (at most) countable disjoint union of open intervals $\left(I_{n}\right)$. Call $E$ a Carleson set if $E$ has measure zero, and

$$
\sum\left|I_{n}\right| \log \left|I_{n}\right|>-\infty
$$

where $\left|I_{n}\right|$ denotes the normalized Lebesgue measure of $I_{n}$. These sets have been studied extensively by Beurling [2], Carleson [3], and others [7], [9], [12] as boundary zero sets for certain spaces of functions holomorphic in the open unit disc. Hayman [5] independently discovered Carleson sets in connection with the Block-Nevanlinna problem (see also [1] and [11]); and H. S. Shapiro [10] found them relevant to the study of the shift operator on certain weighted Bergman spaces.

More precisely, Shapiro studied the operator $M_{z}$ of multiplication by the independent variable $z$ on the Hilbert space $B_{\alpha}$ consisting of all functions holomorphic in the open unit disc and square integrable with respect to the measure $(1-|z|)^{\alpha-1} d x d y \quad(\alpha>0)$. He showed [10, Theorem 1] that if $\mu$ is a positive singular Borel measure on $T$ with modulus of continuity

$$
\omega_{\mu}(\delta)=\sup \{\mu(I):|I|<\delta\}=O(\delta \log (1 / \delta))
$$

(supremum over subarcs $I$ of $T$ ), then the associated singular inner function

$$
S_{\mu}(z)=\exp \int_{0}^{2 \pi}\left(z+e^{i t}\right) /\left(z-e^{i t}\right) d \mu(t)
$$

is cyclic for $M_{z}$; that is, $\operatorname{span}\left\{z^{n} S_{\mu}: n \geqslant 0\right\}$ is dense in $B_{\alpha}$. In the other direction [10, Theorem 2], he showed that $S_{\mu}$ is not cyclic whenever $\mu$ places positive mass on some Carleson set. There are similar results for the Hardy space $H^{p}, 0<p<1$, taken in the weak topology [4, Theorem 13, p. 53].

Received by the editors May 30, 1979.

AMS (MOS) subject classifications (1970). Primary 28A10, 30A78.

Key words and phrases. Hausdorff measure, Carleson set, singular measure, modulus of continuity.

${ }^{1}$ Research partially supported by the National Science Foundation. 
Shapiro's results show that a singular measure $\mu$ with modulus of continuity $O(\delta \log (1 / \delta))$ cannot give positive mass to a Carleson set (a direct proof of this occurs in [8]), and in [10] he indicates without proof that there are measures which fail to have this modulus of continuity, yet still give no mass to any Carleson set.

In this paper we characterize the smoothness classes of measures which place no mass on any Carleson set. Initially the solution is phrased as a result on Hausdorff measures. For convenience we work on the closed unit interval $[0,1]$ rather than the unit circle. Let $h$ be a determining function for a Hausdorff measure $H$ on $[0,1]$; that is, $h$ is nonnegative, continuous, and increasing on $[0,1]$, and for $S$ a subset of $[0,1]$

$$
H(S)=\lim _{r \rightarrow 0+}\left\{\inf \sum_{i} h\left(\left|B_{i}\right|\right)\right\}
$$

where the infimum extends over all coverings $\left\{B_{i}\right\}$ of $S$ by at most countable collections of intervals $B_{i}$ of length $\left|B_{i}\right| \leqslant r$ [6, Chapitre II, §2, p. 23]. Our main result is

Theorem. Suppose $h(t) / t$ is strictly decreasing on $(0,1]$. Then the following conditions on $h$ are equivalent.

(a) $H(E)=0$ for every Carleson set $E \subset[0,1]$.

(b) $\int_{0}^{1} h(t)^{-1} d t=\infty$.

This result, along with standard facts about Hausdorff measure [6, Théoremè III, Chapitre II, p. 27], yields the solution to our original modulus of continuity problem.

COROllary. Suppose $h$ is a determining function with $h(t) / t$ strictly decreasing on $(0,1]$. Then the following statements about $h$ are equivalent.

(a) $\mu(E)=0$ for every Carleson set $E \subset[0,1]$ and every finite positive Borel measure $\mu$ on $[0,1]$ with $\omega_{\mu}(\delta)=O(h(\delta))$.

(b) $\int_{0}^{1} h(t)^{-1} d t=\infty$.

For example, if

$$
\omega_{\mu}(\delta)=O(\delta(\log 1 / \delta)(\log \log 1 / \delta)) \quad(\delta \rightarrow 0+),
$$

then $\mu(E)=0$ for each Carleson set $E$. But for each $\varepsilon>0$ there exists $\mu$ with

$$
\omega_{\mu}(\delta)=O\left(\delta(\log 1 / \delta)(\log \log 1 / \delta)^{1+e}\right) \quad(\delta \rightarrow 0+)
$$

such that $\mu(E)>0$ for some Carleson set $E$.

2. Proof of the Theorem. (b) $\rightarrow$ (a). Suppose (a) fails. Then there is a Carleson set $E$ with $H(E)>0$. We will show that (b) fails, i.e., that $1 / h$ is integrable. It follows from [6, Chapitre II, p. 27, "LEMME"] that inf $\sum h\left(\left|\Delta_{j}\right|\right)=m>0$ where the infimum extends over all at most countable coverings $\left\{\Delta_{j}\right\}$ of $E$ by intervals. Following [6, Chapitre II, p. 29], for $t>0$ we let $E_{t}$ denote the collection of points in $[0,1]$ which lie a distance $<t$ from $E$. Thus $E_{t}$ is an open set which contains $E$. Since $E_{t}$ is a union of open intervals of length $2 t$, it is a disjoint union of finitely many open intervals $\left\{\Delta_{j}\right\}$, each of length $\geqslant 2 t$. Using this disjointness and the fact 
that $h(t) / t$ decreases on $(0,1]$ we obtain

$$
\begin{aligned}
\left|E_{t}\right| & =\sum\left|\Delta_{j}\right|=\sum h\left(\left|\Delta_{j}\right|\right)\left\{\left|\Delta_{j}\right| / h\left(\left|\Delta_{j}\right|\right)\right\} \\
& \geqslant(2 t / h(2 t)) \sum h\left(\left|\Delta_{j}\right|\right) \geqslant 2 t m / h(2 t) .
\end{aligned}
$$

It follows that

$$
\int_{0}^{1 / 2} h(t)^{-1} d t=2 \int_{0}^{1} h(2 t)^{-1} d t \leqslant m^{-1} \int_{0}^{1} t^{-1}\left|E_{t}\right| d t
$$

Since $E$ is a Carleson set, the last integral is finite [3, $§ I$, p. 326, "LEMME”], hence $1 / h$ is integrable. That is, (b) fails.

(a) $\rightarrow$ (b). Suppose (b) fails. Then because $t^{-1} h(t)$ is monotone decreasing on $[0,1]$ we must have

$$
\lim _{t \rightarrow 0+} t^{-1} h(t)=\infty
$$

Now in [6, Chapitre II, §8, p. 30] it is shown that whenever $h(t) / t$ decreases strictly on $(0,1]$ there is a nowhere dense perfect Cantor-type set $E \subset[0,1]$ with $0<H(E)$ $<\infty$. We are going to review the construction of $E$ and show that the integrability of $1 / h$ forces it to be a Carleson set. This will complete the proof.

Construction of $E$. We may suppose without loss of generality that $h(1)=1$. Choose $\lambda_{0}=1$ and, for $n=1,2, \ldots$, choose $0<\lambda_{n}<1$ with $h\left(\lambda_{n}\right)=2^{-n}$. The fact that $t^{-1} h(t)$ decreases strictly on $(0,1]$ insures that

$$
\lambda_{n+1}<\lambda_{n} / 2 \quad(n=0,1,2, \ldots) \text {. }
$$

In particular, $\lambda_{n} \downarrow 0$.

Now divide $[0,1]$ into successive stages of "light" and "dark" intervals, as follows. $E_{0}=[0,1]$ is the unique zeroth stage light intervals. Suppose $E_{n}$ has been obtained as the disjoint union of $2^{n}$ closed light intervals, each of length $\lambda_{n}$. Then $E_{n+1} \subset E_{n}$ is defined to be the union of the $2^{n+1}$ disjoint closed light intervals obtained by deleting from each $n$th stage light interval an open centered dark interval of length

$$
\delta_{n}=\lambda_{n}-2 \lambda_{n+1} .
$$

Inequality (2.2) insures that $\delta_{n}>0$, and that the light and dark intervals at each stage are nontrivial.

Let $E=\bigcap_{n} E_{n}$. Then $E$ is a nowhere dense perfect subset of $[0,1]$ whose complement is the union of all the dark intervals obtained in the course of the construction. By (2.1) we have

$$
|E|=\lim \left|E_{n}\right|=\lim 2^{n} \lambda_{n}=\lim \lambda_{n} / h\left(\lambda_{n}\right)=0 .
$$

By [6, p. 30] we have $0<H(E)<\infty$ (note that this is proved under the additional assumption that $h$ is strictly concave, but all that is actually needed for the proof is (2.2)).

$E$ is a Carleson SET. The complement of $E$ is the disjoint union of the dark intervals. The $n+1$ st stage of the construction contributes $2^{n}$ of these, each of length $\delta_{n}=\lambda_{n}-2 \lambda_{n+1}$. Since $|E|=0$ we have left to check (1.1), which in this 
case is

$$
\sum_{n=0}^{\infty} 2^{n} \delta_{n} \log \left(1 / \delta_{n}\right)<\infty
$$

Note that since $[0,1] \backslash E$ has measure 1 ,

$$
\sum_{n=0}^{\infty} 2^{n} \delta_{n}=1
$$

Since $1 / h$ is assumed to be integrable on $[0,1]$ and $h$ is increasing,

$$
\begin{aligned}
\infty & >\int_{0}^{1} h(t)^{-1} d t=\sum_{n=0}^{\infty} \int_{\lambda_{n+1}}^{\lambda_{n}} h(t)^{-1} d t \\
& >\sum_{n=0}^{\infty} h\left(\lambda_{n}\right)^{-1}\left(\lambda_{n}-\lambda_{n+1}\right)=\sum_{n=0}^{\infty} 2^{n}\left(\lambda_{n}-\lambda_{n+1}\right) \\
& =\sum_{n=0}^{\infty} 2^{n}\left(\delta_{n}+\lambda_{n+1}\right) \text { by (2.3) } \\
& =1+\frac{1}{2} \sum_{n=1}^{\infty} 2^{n} \lambda_{n} \text { by (2.5). }
\end{aligned}
$$

Since $\left|E_{n}\right|=2^{n} \lambda_{n}$, this can be rewritten as

$$
\sum_{n=0}^{\infty}\left|E_{n}\right|<\infty .
$$

Since $\left|E_{n}\right|$ is the sum of the lengths of the dark intervals contained in $E_{n}$-namely those dark intervals from the $n$th stage onward-we have $\left|E_{n}\right|=\sum_{k=n}^{\infty} 2^{k} \delta_{k}$. Substituting this into (2.6) and interchanging the order of summation,

$$
\sum_{k=0}^{\infty}(k+1) 2^{k} \delta_{k}<\infty
$$

To finish the proof, let

$$
A=\left\{n: \delta_{n}<3^{-n}\right\} \text { and } B=\left\{n: \delta_{n}>3^{-n}\right\} .
$$

Since $x \log (1 / x)$ increases on the interval $(0,1 / e]$ we have for all sufficiently large $n \in A$,

$$
2^{n} \delta_{n} \log \left(1 / \delta_{n}\right) \leqslant 2^{n} 3^{-n} \log \left(3^{n}\right)=(\log 3) n(2 / 3)^{n}
$$

so that

$$
\sum_{n \in A} 2^{n} \delta_{n} \log \left(1 / \delta_{n}\right)<\infty
$$

Suppose $n \in B$. Then

$$
\log \left(1 / \delta_{n}\right)<\log \left(3^{n}\right)=n \log 3
$$

so

$$
\sum_{n \in B} 2^{n} \delta_{n} \log \left(1 / \delta_{n}\right)<(\log 3) \sum_{n \in B} n 2^{n} \delta_{n}<\infty,
$$

where the finiteness of the last sum follows from (2.7). Inequalities (2.8) and (2.9) yield (2.4), which completes the proof of the theorem. 
We note in closing that when $1 / h$ is integrable, the Corollary guarantees that there is a Carleson set $E$ and a measure $\mu$ with modulus of continuity $O(h(\delta))$ for which $\mu(E)>0$. In fact the set $E$ constructed in the last part of the proof of the theorem does the job, with $\mu$ the measure induced by the Lebesgue function of $E$ [6, Chapitre II, Théoremè IV, p. 30].

ADDED IN PROOF. After this paper was accepted for publication I learned that its results are contained in Patrick Ahern's recent paper The mean modulus and derivative of an inner function, Indiana Univ. Math. J. 28 (1979), 311-347. In particular Lemma 2.3 and Theorem 2.3 of that paper contain the main result of this one.

An improvement of H. S. Shapiro's result on cyclic inner functions in $B_{\alpha}$ has been obtained by Daniel Leucking in his preprint Cyclic inner functions in the Bergman spaces. Leucking shows that if $\mu$ is a positive singular measure on $T$ that vanishes on all sets of $H$-measure zero, where $H$ has determining function $h(t)=t$ $\log (1 / t)$, then $S_{\mu}$ is cyclic in $B_{\alpha}$. In the same paper he obtains a factorization of singular inner functions into cyclic and purely noncyclic parts.

\section{REFERENCES}

1. P. Ahern, An example in the theory of functions (preprint).

2. A. Beurling, Ensembles exceptionnels, Acta Math. 72 (1940), 1-13.

3. L. Carleson, Sets of uniqueness for functions regular in the unit circle, Acta Math. 87 (1952), 325-345.

4. P. L. Duren, B. W. Romberg and A. L. Shields, Linear functionals on $H^{p}$ spaces with $0<p<1$, J. Reine Angew. Math. 238 (1969), 32-60.

5. W. Hayman, On Nevanlinna's second theorem and extensions, Rend. Circ. Mat. Palermo (2) 2 (1953), 346-392.

6. J.-P. Kahane and R. Salem, Ensembles parfaits et séries trigonométriques, Hermann, Paris, 1963.

7. J. D. Nelson, $A$ characterization of zero sets for $A^{\infty}$, Michigan Math. J. 18 (1971), 141-147.

8. D. J. Newman, J. T. Schwartz and H. S. Shapiro, On generators of the Banach algebras $l_{1}$ and $L_{1}(0, \infty)$, Trans. Amer. Math. Soc. 107 (1963), 466-484.

9. W. P. Novinger, Holomorphic functions with infinitely differentiable boundary values, Illinois J. Math. 15 (1971), 80-90.

10. H. S. Shapiro, Some remarks on weighted polynomial approximations of holomorphic functions, Mat. Sb. 2 (1967), 285-294.

11. J. H. Shapiro, A characterization by Cauchy transforms of the Beurling-Carleson-Hayman thin sets, Michigan Math. J. (to appear).

12. B. A. Taylor and D. L. Williams, Ideals in rings of analytic functions with smooth boundary values, Canad. J. Math. 22 (1970), 1266-1283.

Department of Mathematics, Michigan State University, East Lansing, Michigan 48824 